\title{
A Nonsmooth Optimization Approach for Hemivariational Inequalities with Applications to Contact Mechanics
}

\author{
Michal Jureczka ${ }^{1} \cdot$ Anna Ochal ${ }^{1}$ (D)
}

Published online: 13 July 2019

(c) The Author(s) 2019

\begin{abstract}
In this paper we introduce an abstract nonsmooth optimization problem and prove existence and uniqueness of its solution. We present a numerical scheme to approximate this solution. The theory is later applied to a sample static contact problem describing an elastic body in frictional contact with a foundation. This contact is governed by a nonmonotone friction law with dependence on normal and tangential components of displacement. Finally, computational simulations are performed to illustrate obtained results.
\end{abstract}

Keywords Nonmonotone friction - Optimization problem · Error estimate · Finite element method $\cdot$ Numerical simulations

Mathematics Subject Classification 35Q74 - 49J40 - 65K10 - 65M60 - 74S05 . $74 \mathrm{M} 15 \cdot 74 \mathrm{M} 10 \cdot 74 \mathrm{G} 15$

\section{Introduction}

In the literature we can find examples of many models describing deformation of a body that is partly in contact with another object, the so-called foundation. In various contact models boundary conditions enforced on the part of the body contacting the foundation appear. Functions that occur in these conditions model response of the foundation in the normal direction to the contact boundary and in tangential direction to the boundary (friction law). In many cases these functions are monotone, such as when Coulomb's law of dry friction is considered, but in applications this may not

Anna Ochal

anna.ochal@uj.edu.pl

Michal Jureczka

michal.jureczka@uj.edu.pl

1 Faculty of Mathematics and Computer Science, Jagiellonian University in Krakow, Lojasiewicza 6, 30-348 Krakow, Poland 
always be the case. What is more, the friction bound may change as the penetration of the foundation by body increases. Nonmonotonicity of functions describing contact laws and influence of normal displacement of the body on friction law cause some difficulties in analytical and numerical treatment of considered problems.

In this paper we introduce an abstract framework that can be used to numerically approximate a solution to a class of mechanical contact problems. We present a nonsmooth optimization problem and prove existence and uniqueness of a solution to this problem. Next we present a numerical scheme approximating this solution and provide numerical error estimate. We apply this theory to a static contact problem describing an elastic body in contact with a foundation. This contact is governed by a nonmonotone friction law with dependence on normal and tangential components of displacement. Weak formulation of introduced contact problem is presented in the form of hemivariational inequality. In the end we show results of computational simulations and describe the numerical algorithm that was used to obtain these results.

Let us now briefly present references in the literature. The definition and properties of Clarke subdifferential and tools used to solve optimization problems were introduced in [6]. Comparison of nonsmooth and nonconvex optimization methods can be found in [1], and details on computational contact mechanics is presented in [19]. The theory of hemivariational inequalities was developed in [17], and the idea to use Finite Element Method to solve these inequalities was presented in [13]. Another early study of vector-valued hemivariational problems in the context of FEM can be found in [14]. More recent analysis of hemivariational and variational-hemivariational inequalities was presented in $[10,15,16]$, whereas numerical analysis of such problems can be found for example in papers [2-4,8,9,11,12].

A similar mechanical model to the one described in the paper was already considered in [15], where the authors prove only existence of a solution using surjectivity result for pseudomonotone, coercive multifunction without requiring any smallness assumption.

An error estimate concerning stationary variational-hemivariational inequalities was presented in [8]. In our case variational part of inequality is not present and the inequality is not constrained, however error estimate had to be generalized to reflect dependence of friction law on normal component of the displacement.

A numerical treatment of mechanical problem leading to hemivariational inequality using two approaches - nonsmooth and nonconvex optimization and quasi-augmented Lagrangian method is presented in [2]. As the smallness assumption is not required, this once again does not guarantee uniqueness and leads to a nonconvex optimization problem. There, the authors assume contact to be bilateral and consider friction law which does not depend on normal component of the displacement.

This paper is organized as follows. Section 2 contains a general differential inclusion problem and an optimization problem. We show that under introduced assumptions both problems are equivalent and have a unique solution. In Sect. 3 we proceed with a discrete scheme that approximates solution to introduced optimization problem and we prove theorem concerning numerical error estimate. An application of presented theory in the form of mechanical contact model is indicated in Sect. 4, along with its weak formulation. Finally, in Sect. 5, we describe computational algorithm used to solve mechanical contact problem and present simulations for a set of sample data. 


\section{A General Optimization Problem}

Let us start with basic notation used in this paper. For a normed space $X$, we denote by $\|\cdot\|_{X}$ its norm, by $X^{*}$ its dual space and by $\langle\cdot, \cdot\rangle_{X^{*} \times X}$ the duality pairing of $X^{*}$ and $X$. By $c>0$ we denote a generic constant (value of $c$ may differ in different equations).

Let us now assume that $j: X \rightarrow \mathbb{R}$ is locally Lipschitz continuous. The generalized directional derivative of $j$ at $x \in X$ in the direction $v \in X$ is defined by

$$
j^{0}(x ; v):=\limsup _{y \rightarrow x, \lambda \searrow 0} \frac{j(y+\lambda v)-j(y)}{\lambda} .
$$

The generalized subdifferential of $j$ at $x$ is a subset of the dual space $X^{*}$ given by

$$
\partial j(x):=\left\{\xi \in X^{*} \mid\langle\xi, v\rangle_{X^{*} \times X} \leq j^{0}(x ; v) \text { for all } v \in X\right\}
$$

If $j: X^{n} \rightarrow \mathbb{R}$ is a locally Lipschitz function of $n$ variables, then we denote by $\partial_{i} j$ and $j_{i}^{0}$ the Clarke subdifferential and generalized directional derivative with respect to $i$-th variable of $j$, respectively.

Let now $V$ be a reflexive Banach space and $X$ be a Banach space. Let $\gamma \in \mathcal{L}(V, X)$ be linear and continuous operator from $V$ to $X$, and $c_{\gamma}:=\|\gamma\|_{\mathcal{L}(V, X)}$. We denote by $\gamma^{*}: X^{*} \rightarrow V^{*}$ the adjoint operator to $\gamma$. Let $A: V \rightarrow V^{*}, J: X \times X \rightarrow \mathbb{R}$ and $f \in V^{*}$. We formulate the operator inclusion problem as follows.

Problem $\boldsymbol{P}_{\text {incl }}$ Find $u \in V$ such that

$$
A u+\gamma^{*} \partial_{2} J(\gamma u, \gamma u) \ni f
$$

In the study of Problem $P_{\text {incl }}$ we make the following assumptions.

$\underline{H(A)}$ : The operator $A: V \rightarrow V^{*}$ is such that

(a) $A$ is linear and bounded,

(b) $A$ is symmetric, i.e. $\langle A u, v\rangle_{V^{*} \times V}=\langle A v, u\rangle_{V^{*} \times V}$ for all $u, v \in V$,

(c) there exists $m_{A}>0$ such that $\langle A u, u\rangle_{V^{*} \times V} \geq m_{A}\|u\|_{V}^{2}$ for all $u \in V$.

$H(J):$ The functional $J: X \times X \rightarrow \mathbb{R}$ satisfies

(a) $J$ is locally Lipschitz continuous with respect to its second variable,

(b) there exist $c_{0}, c_{1}, c_{2} \geq 0$ such that

$$
\left\|\partial_{2} J(w, v)\right\|_{X^{*}} \leq c_{0}+c_{1}\|v\|_{X}+c_{2}\|w\|_{X} \quad \text { for all } w, v \in X
$$

(c) there exist $m_{\alpha}, m_{L} \geq 0$ such that

$$
\begin{aligned}
& J_{2}^{0}\left(w_{1}, v_{1} ; v_{2}-v_{1}\right)+J_{2}^{0}\left(w_{2}, v_{2} ; v_{1}-v_{2}\right) \\
& \quad \leq m_{\alpha}\left\|v_{1}-v_{2}\right\|_{X}^{2}+m_{L}\left\|w_{1}-w_{2}\right\|_{X}\left\|v_{1}-v_{2}\right\|_{X}
\end{aligned}
$$

for all $w_{1}, w_{2}, v_{1}, v_{2} \in X$. 
$H(f): \quad f \in V^{*}$.

$\overline{\left(H_{S}\right)}: \quad m_{A}>\left(2 m_{\alpha}+m_{L}\right) c_{\gamma}^{2}$.

We remark that $H(J)(\mathrm{c})$ is equivalent to the following condition

$$
\begin{aligned}
& \left\langle\partial_{2} J\left(w_{1}, v_{1}\right)-\partial_{2} J\left(w_{2}, v_{2}\right), v_{1}-v_{2}\right\rangle_{X^{*} \times X} \\
& \quad \geq-m_{\alpha}\left\|v_{1}-v_{2}\right\|_{X}^{2}-m_{L}\left\|w_{1}-w_{2}\right\|_{X}\left\|v_{1}-v_{2}\right\|_{X}
\end{aligned}
$$

for all $w_{1}, w_{2}, v_{1}, v_{2} \in X$. Moreover, in a special case when $J$ does not depend on its first variable, condition $H(J)(\mathrm{c})$ holds with $m_{L}=0$ and we obtain the well known relaxed monotonicity condition, i.e. for all $v_{1}, v_{2} \in X$

$$
\left\langle\partial J\left(v_{1}\right)-\partial J\left(v_{2}\right), v_{1}-v_{2}\right\rangle_{X^{*} \times X} \geq-m_{\alpha}\left\|v_{1}-v_{2}\right\|_{X}^{2} .
$$

So, condition $H(J)(c)$ is more general than (2.2).

We start with a uniqueness result for Problem $P_{i n c l}$, provided that a solution exists.

Lemma 1 Assume that $H(A), H(J), H(f)$ and $\left(H_{S}\right)$ hold. If Problem $P_{\text {incl }}$ has a solution $u \in V$, then it is unique and satisfies

$$
\|u\|_{V} \leq c\left(1+\|f\|_{V^{*}}\right)
$$

with a positive constant $c$.

Proof Let $u \in V$ be a solution to Problem $P_{\text {incl }}$. This means that there exists $z \in$ $\partial_{2} J(\gamma u, \gamma u)$ such that

$$
A u+\gamma^{*} z=f
$$

From the definition of generalized directional derivative of $J(\gamma u, \cdot)$ we have for all $v \in V$

$$
\langle f-A u, v\rangle_{V^{*} \times V}=\left\langle\gamma^{*} z, v\right\rangle_{V^{*} \times V}=\langle z, \gamma v\rangle_{X^{*} \times X} \leq J_{2}^{0}(\gamma u, \gamma u ; \gamma v) .
$$

Let us now assume that Problem $P_{\text {incl }}$ has two different solutions $u_{1}$ and $u_{2}$. For a solution $u_{1}$ we set $v=u_{2}-u_{1}$ in (2.4) to get

$$
\left\langle f, u_{2}-u_{1}\right\rangle_{V^{*} \times V}-\left\langle A u_{1}, u_{2}-u_{1}\right\rangle_{V^{*} \times V} \leq J_{2}^{0}\left(\gamma u_{1}, \gamma u_{1} ; \gamma u_{2}-\gamma u_{1}\right) .
$$

For a solution $u_{2}$ we set $v=u_{1}-u_{2}$ in (2.4) to get

$$
\left\langle f, u_{1}-u_{2}\right\rangle_{V^{*} \times V}-\left\langle A u_{2}, u_{1}-u_{2}\right\rangle_{V^{*} \times V} \leq J_{2}^{0}\left(\gamma u_{2}, \gamma u_{2} ; \gamma u_{1}-\gamma u_{2}\right) .
$$

Adding the above inequalities, we obtain

$$
\begin{aligned}
& \left\langle A u_{1}-A u_{2}, u_{1}-u_{2}\right\rangle_{V^{*} \times V} \\
& \quad \leq J_{2}^{0}\left(\gamma u_{1}, \gamma u_{1} ; \gamma u_{2}-\gamma u_{1}\right)+J_{2}^{0}\left(\gamma u_{2}, \gamma u_{2} ; \gamma u_{1}-\gamma u_{2}\right) .
\end{aligned}
$$


Hence, $H(A)(\mathrm{c})$ and $H(J)(\mathrm{c})$ yield

$$
m_{A}\left\|u_{1}-u_{2}\right\|_{V}^{2} \leq\left(m_{\alpha}+m_{L}\right)\left\|\gamma u_{1}-\gamma u_{2}\right\|_{X}^{2},
$$

and finally

$$
\left(m_{A}-\left(m_{\alpha}+m_{L}\right) c_{\gamma}^{2}\right)\left\|u_{1}-u_{2}\right\|_{V}^{2} \leq 0
$$

Under assumption $\left(H_{S}\right)$, we obtain that if Problem $P_{\text {incl }}$ has a solution, it is unique.

Now, in order to prove (2.3), we set $v=-u$ in (2.4) to obtain

$$
\langle A u, u\rangle_{V^{*} \times V} \leq J_{2}^{0}(\gamma u, \gamma u ;-\gamma u)+\langle f, u\rangle_{V^{*} \times V}
$$

Using $H(J)(\mathrm{b})$ and (c), we get

$$
\begin{aligned}
& J_{2}^{0}(\gamma u, \gamma u ;-\gamma u) \leq\left(m_{\alpha}+m_{L}\right)\|\gamma u\|_{X}^{2}-J_{2}^{0}(0,0 ; \gamma u) \\
& \quad \leq\left(m_{\alpha}+m_{L}\right)\|\gamma u\|_{X}^{2}+c_{0}\|\gamma u\|_{X} .
\end{aligned}
$$

Combining (2.5) and (2.6), we have

$$
m_{A}\|u\|_{V}^{2} \leq\left(m_{\alpha}+m_{L}\right)\|\gamma u\|_{X}^{2}+c_{0}\|\gamma u\|_{X}+\|f\|_{V^{*}}\|u\|_{V}
$$

and

$$
\left(m_{A}-\left(m_{\alpha}+m_{L}\right) c_{\gamma}^{2}\right)\|u\|_{V} \leq c\left(1+\|f\|_{V^{*}}\right)
$$

From $\left(H_{S}\right)$ we obtain required estimate.

We now consider an optimization problem, which will be equivalent to Problem $P_{\text {incl }}$ under introduced assumptions. To this end, let the functional $\mathcal{L}: V \times V \rightarrow \mathbb{R}$ be defined for all $w, v \in V$ as follows

$$
\mathcal{L}(w, v)=\frac{1}{2}\langle A v, v\rangle_{V^{*} \times V}-\langle f, v\rangle_{V^{*} \times V}+J(\gamma w, \gamma v)
$$

The next lemma collects some properties of the functional $\mathcal{L}$.

Lemma 2 Under assumptions $H(A), H(J), H(f)$ and $\left(H_{s}\right)$, the functional $\mathcal{L}: V \times$ $V \rightarrow \mathbb{R}$ defined by (2.7) satisfies

(i) $\mathcal{L}(w, \cdot)$ is locally Lipschitz continuous for all $w \in V$,

(ii) $\partial_{2} \mathcal{L}(w, v) \subseteq A v-f+\gamma^{*} \partial_{2} J(\gamma w, \gamma v)$ for all $w, v \in V$,

(iii) $\mathcal{L}(w, \cdot)$ is strictly convex for all $w \in V$,

(iv) $\mathcal{L}(w, \cdot)$ is coercive for all $w \in V$. 
Proof The proof of $(i)$ is immediate since for a fixed $w \in V$ the functional $\mathcal{L}(w, \cdot)$ is locally Lipschitz continuous as a sum of locally Lipschitz continuous functions with respect to $v$.

For the proof of $(i i)$, we observe that from $H(A)$ and $H(f)$, the functions

$$
f_{1}: V \ni v \mapsto \frac{1}{2}\langle A v, v\rangle_{V^{*} \times V} \in \mathbb{R}, \quad f_{2}: V \ni v \mapsto\langle f, v\rangle_{V^{*} \times V} \in \mathbb{R}
$$

are strictly differentiable and

$$
f_{1}^{\prime}(v)=A v, \quad f_{2}^{\prime}(v)=f .
$$

Now, using the chain rule for generalized subgradient (cf. Propositions 3.35 and 3.37 in [15]), we obtain

$$
\begin{aligned}
\partial_{2} \mathcal{L}(w, v) & =f_{1}^{\prime}(v)-f_{2}^{\prime}(v)+\partial_{2}(J \circ \gamma)(\gamma w, v) \\
& \subseteq A v-f+\gamma^{*} \partial_{2} J(\gamma w, \gamma v),
\end{aligned}
$$

which concludes $(i i)$.

To prove (iii), first, we will show that for any fixed $w \in V$, the operator $v \mapsto$ $\partial_{2} \mathcal{L}(w, v)$ is strongly monotone. Then, we will use Theorem 3.4 in [7] to deduce that $\mathcal{L}(w, \cdot)$ is strongly convex for all $w \in V$. Finally, the latter implies condition (iii). Hence, it remains to show that $v \mapsto \partial_{2} \mathcal{L}(w, v)$ is strongly monotone. To this end, let us fix $w, v_{i} \in V$ with $i=1,2$. We take $\zeta_{i} \in \partial_{2} \mathcal{L}\left(w, v_{i}\right)$. From (ii) there exist $z_{i} \in \partial_{2} J\left(\gamma w, \gamma v_{i}\right)$ such that

$$
\zeta_{i}=A v_{i}-f+\gamma^{*} z_{i}
$$

Hence, using $H(A)(\mathrm{c})$ and (2.2), we obtain

$$
\begin{aligned}
& \left\langle\zeta_{1}-\zeta_{2}, v_{1}-v_{2}\right\rangle_{V^{*} \times V} \\
& \quad=\left\langle A v_{1}-A v_{2}, v_{1}-v_{2}\right\rangle_{V^{*} \times V}+\left\langle\gamma^{*} z_{1}-\gamma^{*} z_{2}, v_{1}-v_{2}\right\rangle_{V^{*} \times V} \\
& \quad \geq m_{A}\left\|v_{1}-v_{2}\right\|_{V}^{2}+\left\langle z_{1}-z_{2}, \gamma v_{1}-\gamma v_{2}\right\rangle_{X^{*} \times X} \\
& \quad \geq m_{A}\left\|v_{1}-v_{2}\right\|_{V}^{2}-m_{\alpha}\left\|\gamma v_{1}-\gamma v_{2}\right\|_{X}^{2} \\
& \quad \geq\left(m_{A}-m_{\alpha} c_{\gamma}^{2}\right)\left\|v_{1}-v_{2}\right\|_{V}^{2} .
\end{aligned}
$$

From $\left(H_{s}\right)$ we see that $\partial_{2} \mathcal{L}(w, \cdot)$ is strongly monotone for every $w \in V$. For the proof of $(i v)$, let us fix $w, v \in V$. From $H(A)(\mathrm{c})$ and $H(f)$ we obtain

$$
\mathcal{L}(w, v) \geq \frac{1}{2} m_{A}\|v\|_{V}^{2}-\|f\|_{V^{*}}\|v\|_{V}+J(\gamma w, \gamma v)
$$


Now, using the Lebourg mean value theorem (cf. Proposition 3.36 in [15]), we get that there exists $\lambda \in(0,1)$ and $\eta \in \partial_{2}(J \circ \gamma)(\gamma w, \lambda v)$ such that

$$
J(\gamma w, \gamma v)=\langle\eta, v\rangle_{V^{*} \times V}+J(\gamma w, 0) .
$$

Since $\partial_{2}(J \circ \gamma)(\gamma w, \lambda v) \subseteq \gamma^{*} \partial_{2} J(\gamma w, \lambda \gamma v)$ we have $\eta \in \gamma^{*} \partial_{2} J(\gamma w, \lambda \gamma v)$. Then there exists $z_{1} \in \partial_{2} J(\gamma w, \lambda \gamma v)$ such that $\eta=\gamma^{*} z_{1}$. Taking $z_{2} \in \partial_{2} J(\gamma w, 0)$ and by (2.1), we obtain

$\lambda\left\langle\gamma^{*} z_{1}-\gamma^{*} z_{2}, v\right\rangle_{V^{*} \times V}=\left\langle z_{1}-z_{2}, \lambda \gamma v\right\rangle_{X^{*} \times X} \geq-m_{\alpha}\|\lambda \gamma v\|_{X}^{2} \geq-m_{\alpha} \lambda^{2} c_{\gamma}^{2}\|v\|_{V}^{2}$,

and this, along with the fact that $\lambda \in(0,1)$, leads to

$$
\langle\eta, v\rangle_{V^{*} \times V} \geq-m_{\alpha} c_{\gamma}^{2}\|v\|_{V}^{2}+\left\langle z_{2}, \gamma v\right\rangle_{X^{*} \times X}
$$

Using $H(J)(\mathrm{b})$, we get

$$
\left\langle z_{2}, \gamma v\right\rangle_{X^{*} \times X} \geq-\left|\left\langle z_{2}, \gamma v\right\rangle_{X^{*} \times X}\right| \geq-\left\|\partial_{2} J(\gamma w, 0)\right\|_{X^{*}}\|\gamma v\|_{X} \geq-c\left(1+\|w\|_{V}\right)\|v\|_{V} .
$$

Combining (2.8)-(2.11) and because $J(\gamma w, 0)$ is bounded from below for fixed $w$, we get

$$
\mathcal{L}(w, v) \geq\left(\frac{1}{2} m_{A}-m_{\alpha} c_{\gamma}^{2}\right)\|v\|_{V}^{2}-c\|v\|_{V}-c .
$$

From $\left(H_{S}\right)$ we see that $\mathcal{L}(w, \cdot)$ is coercive for every $w \in V$.

The problem under consideration reads as follows.

Problem $\boldsymbol{P}_{\text {opt }}$ Find $u \in V$ such that

$$
0 \in \partial_{2} \mathcal{L}(u, u)
$$

We are now in a position to prove the existence and uniqueness result for the above optimization problem.

Lemma 3 Assume that $H(A), H(J), H(f)$ and $\left(H_{S}\right)$ hold. Then Problem $P_{\text {opt }}$ has a unique solution $u \in V$.

Proof We introduce operator $\Lambda: V \rightarrow V$ defined for all $w \in V$ as follows

$$
\Lambda w=\underset{v \in V}{\arg \min } \mathcal{L}(w, v) .
$$

From Lemma 2(i), (iv) we see that for fixed $w$ functional $\mathcal{L}(w, \cdot)$ is proper, lower semicontinuous and coercive. This implies that it attains a global minimum. Uniqueness of that minimum is guaranteed by Lemma 2(iii). We can conclude that operator 
$\Lambda$ is well defined. Now we prove that it is a contraction. Take $u_{i} \in V$ for $i=1,2$ and let $\widehat{u}_{i}=\Lambda u_{i}$. Because of strict convexity of $\mathcal{L}(w, \cdot)$, we have

$$
\widehat{u}_{i}=\underset{v \in V}{\arg \min } \mathcal{L}\left(u_{i}, v\right) \quad \text { if and only if } \quad 0 \in \partial_{2} \mathcal{L}\left(u_{i}, \widehat{u}_{i}\right)
$$

(see Theorem 1.23 in [13]). From similar arguments to those used in proofs of Lemmata 1 and 2 with fixed first argument of functional $\mathcal{L}$, we have for all $v \in V$

$$
\left\langle f-A \widehat{u}_{i}, v\right\rangle_{V^{*} \times V} \leq J_{2}^{0}\left(\gamma u_{i}, \gamma \widehat{u}_{i} ; \gamma v\right)
$$

Taking for $i=1$ value $v=\widehat{u}_{2}-\widehat{u}_{1}$, for $i=2$ value $v=\widehat{u}_{1}-\widehat{u}_{2}$ and adding these inequalities, we obtain

$$
\begin{aligned}
& \left\langle A \widehat{u}_{1}-A \widehat{u}_{2}, \widehat{u}_{1}-\widehat{u}_{2}\right\rangle_{V^{*} \times V} \\
& \quad \leq J_{2}^{0}\left(\gamma u_{1}, \gamma \widehat{u}_{1} ; \gamma \widehat{u}_{2}-\gamma \widehat{u}_{1}\right)+J_{2}^{0}\left(\gamma u_{2}, \gamma \widehat{u}_{2} ; \gamma \widehat{u}_{1}-\gamma \widehat{u}_{2}\right) .
\end{aligned}
$$

From assumptions $H(A)(\mathrm{c})$ and $H(J)(\mathrm{c})$, we get

$$
m_{A}\left\|\widehat{u}_{1}-\widehat{u}_{2}\right\|_{V}^{2} \leq m_{\alpha}\left\|\gamma \widehat{u}_{1}-\gamma \widehat{u}_{2}\right\|_{X}^{2}+m_{L}\left\|\gamma u_{1}-\gamma u_{2}\right\|_{X}\left\|\gamma \widehat{u}_{1}-\gamma \widehat{u}_{2}\right\|_{X} .
$$

Using the elementary inequality $a b \leq \frac{a^{2}}{2}+\frac{b^{2}}{2}$, we obtain

$$
m_{A}\left\|\widehat{u}_{1}-\widehat{u}_{2}\right\|_{V}^{2} \leq m_{\alpha} c_{\gamma}^{2}\left\|\widehat{u}_{1}-\widehat{u}_{2}\right\|_{V}^{2}+\frac{m_{L} c_{\gamma}^{2}}{2}\left(\left\|u_{1}-u_{2}\right\|_{V}^{2}+\left\|\widehat{u}_{1}-\widehat{u}_{2}\right\|_{V}^{2}\right) .
$$

Because of $\left(H_{S}\right)$, we can rearrange these terms to get

$$
\left\|\widehat{u}_{1}-\widehat{u}_{2}\right\|_{V}^{2} \leq \frac{m_{L} c_{\gamma}^{2}}{2 m_{A}-2 m_{\alpha} c_{\gamma}^{2}-m_{L} c_{\gamma}^{2}}\left\|u_{1}-u_{2}\right\|_{V}^{2}
$$

Using assumption $\left(H_{S}\right)$ once more, we obtain that the operator $\Lambda$ is a contraction. From the Banach fixed point theorem we know that there exists a unique $u^{*} \in V$ such that $\Lambda u^{*}=u^{*}$, so $0 \in \partial_{2} \mathcal{L}\left(u^{*}, u^{*}\right)$.

Let us conclude the results from Lemmata 1, 2 and 3 in the following theorem.

Theorem 4 Assume that $H(A), H(J), H(f)$ and $\left(H_{S}\right)$ hold. Then Problems $P_{\text {incl }}$ and $P_{\text {opt }}$ are equivalent, they have a unique solution $u \in V$ and this solution satisfies

$$
\|u\|_{V} \leq c\left(1+\|f\|_{V^{*}}\right)
$$

with a positive constant $c$. 
Proof Lemma 2(ii) implies that every solution to Problem $P_{\text {opt }}$ solves Problem $P_{\text {incl }}$. Using this fact, Lemmata 1 and 3 we see that a unique solution to Problem $P_{\text {opt }}$ is also a unique solution to Problem $P_{\text {incl }}$. Because of the uniqueness of the solution to Problem $P_{i n c l}$ we get that Problems $P_{i n c l}$ and $P_{\text {opt }}$ are equivalent. The estimate in the statement of the theorem follows from Lemma 1.

\section{Numerical Scheme}

Let $V^{h} \subset V$ be a finite dimensional subspace with a discretization parameter $h>0$. We present the following discrete scheme of Problem $P_{\text {opt }}$.

Problem $\boldsymbol{P}_{\text {opt }}^{\boldsymbol{h}}$ Find $u^{h} \in V^{h}$ such that

$$
0 \in \partial_{2} \mathcal{L}\left(u^{h}, u^{h}\right)
$$

We remark that existence of a unique solution to Problem $P_{o p t}^{h}$ and equivalence to the discrete version of Problem $P_{\text {incl }}$ follow from application of Theorem 4 in this new setting. Now let us present the following main theorem concerning error estimatie of introduced numerical scheme.

Theorem 5 Assume that $H(A), H(J), H(f)$ and $\left(H_{S}\right)$ hold. Then for the unique solutions $u$ and $u^{h}$ to Problems $P_{\text {opt }}$ and $P_{o p t}^{h}$, respectively, there exists a constant $c>0$ such that

$$
\left\|u-u^{h}\right\|_{V}^{2} \leq c \inf _{v^{h} \in V^{h}}\left\{\left\|u-v^{h}\right\|_{V}^{2}+\left\|\gamma u-\gamma v^{h}\right\|_{X}+R\left(u, v^{h}\right)\right\}
$$

where a residual quantity is given by

$$
R\left(u, v^{h}\right)=\left\langle A u, v^{h}-u\right\rangle_{V^{*} \times V}+\left\langle f, u-v^{h}\right\rangle_{V^{*} \times V} .
$$

Proof Let $u$ be a solution to Problem $P_{o p t}$ and $u^{h}$ be a solution to Problem $P_{o p t}^{h}$. Then they are solutions to corresponding inclusion problems and satisfy respectively

$$
\begin{gathered}
\langle f-A u, v\rangle_{V^{*} \times V} \leq J_{2}^{0}(\gamma u, \gamma u ; \gamma v) \text { for all } v \in V \\
\left\langle f-A u^{h}, v\right\rangle_{V^{*} \times V} \leq J_{2}^{0}\left(\gamma u^{h}, \gamma u^{h} ; \gamma v\right) \text { for all } v \in V^{h} .
\end{gathered}
$$

Taking (3.3) with $v=u^{h}-u$, and (3.4) with $v=v^{h}-u^{h}$, then adding these inequalities, we obtain for all $v^{h} \in V^{h}$

$$
\begin{aligned}
& \left\langle f, v^{h}-u\right\rangle_{V^{*} \times V}+\left\langle A u^{h}-A u, u^{h}-u\right\rangle_{V^{*} \times V}-\left\langle A u^{h}, v^{h}-u\right\rangle_{V^{*} \times V} \\
& \quad \leq J_{2}^{0}\left(\gamma u, \gamma u ; \gamma u^{h}-\gamma u\right)+J_{2}^{0}\left(\gamma u^{h}, \gamma u^{h} ; \gamma v^{h}-\gamma u^{h}\right) .
\end{aligned}
$$


We observe that by subadditivity of generalized directional derivative (cf. [15], Proposition 3.23(i)) and $H(J)(\mathrm{c})$, we have

$$
\begin{aligned}
& J_{2}^{0}\left(\gamma u, \gamma u ; \gamma u^{h}-\gamma u\right)+J_{2}^{0}\left(\gamma u^{h}, \gamma u^{h} ; \gamma v^{h}-\gamma u^{h}\right) \\
& \quad \leq J_{2}^{0}\left(\gamma u, \gamma u ; \gamma u^{h}-\gamma u\right)+J_{2}^{0}\left(\gamma u^{h}, \gamma u^{h} ; \gamma u-\gamma u^{h}\right)+J_{2}^{0}\left(\gamma u^{h}, \gamma u^{h} ; \gamma v^{h}-\gamma u\right) \\
& \quad \leq\left(m_{\alpha}+m_{L}\right)\left\|\gamma u^{h}-\gamma u\right\|_{X}^{2}+\left(c_{0}+\left(c_{1}+c_{2}\right)\left\|\gamma u^{h}\right\|_{X}\right)\left\|\gamma v^{h}-\gamma u\right\|_{X} .
\end{aligned}
$$

From the statement of Lemma 1 applied to discrete version of Problem $P_{\text {incl }}$ we get that $\left\|\gamma u^{h}\right\|_{X} \leq c_{\gamma}\left\|u^{h}\right\|_{V} \leq c\left(1+\|f\|_{V^{*}}\right)$ is uniformly bounded with respect to $h$. Hence, returning to (3.5) and using (3.6), we obtain for all $v^{h} \in V^{h}$

$$
\begin{aligned}
& \left\langle A u^{h}-A u, u^{h}-u\right\rangle_{V^{*} \times V} \leq\left\langle A u^{h}-A u, v^{h}-u\right\rangle_{V^{*} \times V}+\left\langle A u, v^{h}-u\right\rangle_{V^{*} \times V} \\
& \quad+\left\langle f, u-v^{h}\right\rangle_{V^{*} \times V}+\left(m_{\alpha}+m_{L}\right) c_{\gamma}^{2}\left\|u^{h}-u\right\|_{V}^{2}+c\left\|\gamma v^{h}-\gamma u\right\|_{X} .
\end{aligned}
$$

By assumption $H(A)$ and definition (3.2), we get for all $v^{h} \in V^{h}$

$$
\begin{gathered}
m_{A}\left\|u^{h}-u\right\|_{V}^{2} \leq c\left\|u^{h}-u\right\|_{V}\left\|v^{h}-u\right\|_{V}+R\left(u, v^{h}\right) \\
+\left(m_{\alpha}+m_{L}\right) c_{\gamma}^{2}\left\|u-u^{h}\right\|_{V}^{2}+c\left\|\gamma u-\gamma v^{h}\right\|_{X} .
\end{gathered}
$$

Finally, the elementary inequality $a b \leq \varepsilon a^{2}+\frac{b^{2}}{4 \varepsilon}$ with $\varepsilon>0$ yields

$$
\begin{aligned}
& m_{A}\left\|u-u^{h}\right\|_{V}^{2} \leq \varepsilon\left\|u-u^{h}\right\|_{V}^{2}+\frac{c^{2}}{4 \varepsilon}\left\|u-v^{h}\right\|_{V}^{2}+R\left(u, v^{h}\right) \\
& +\left(m_{\alpha}+m_{L}\right) c_{\gamma}^{2}\left\|u-u^{h}\right\|_{V}^{2}+c\left\|\gamma u-\gamma v^{h}\right\|_{X} .
\end{aligned}
$$

This is equivalent for all $v^{h} \in V^{h}$ to

$$
\left(m_{A}-\left(m_{\alpha}+m_{L}\right) c_{\gamma}^{2}-\varepsilon\right)\left\|u-u^{h}\right\|_{V}^{2} \leq \frac{c}{\varepsilon}\left\|u-v^{h}\right\|_{V}^{2}+R\left(u, v^{h}\right)+c\left\|\gamma u-\gamma v^{h}\right\|_{X} .
$$

Taking sufficiently small $\varepsilon$ and using $\left(H_{S}\right)$ we obtain the desired conclusion.

\section{Application to Contact Mechanics}

In this section we apply the results of previous sections to a sample mechanical contact problem. Let us start by introducing the physical setting and notation.

An elastic body occupies a domain $\Omega \subset \mathbb{R}^{d}$, where $d=2,3$ in application. We assume that its boundary $\Gamma$ is divided into three disjoint measurable parts: $\Gamma_{D}, \Gamma_{C}, \Gamma_{N}$, where the part $\Gamma_{D}$ has a positive measure. Additionally $\Gamma$ is Lipschitz continuous, and therefore the outward normal vector $\boldsymbol{v}$ to $\Gamma$ exists a.e. on the boundary. The body is clamped on $\Gamma_{D}$, i.e. its displacement is equal to $\mathbf{0}$ on this part of boundary. A surface force of density $\boldsymbol{f}_{N}$ acts on the boundary $\Gamma_{N}$ and a body force of density $\boldsymbol{f}_{0}$ acts in $\Omega$. 
The contact phenomenon on $\Gamma_{C}$ is modeled using general subdifferential inclusions. We are interested in finding the displacement of the body in a static state.

Let us denote by "." and $\|\cdot\|$ the scalar product and the Euclidean norm in $\mathbb{R}^{d}$ or $\mathbb{S}^{d}$, respectively, where $\mathbb{S}^{d}=\mathbb{R}_{s y m}^{d \times d}$. Indices $i$ and $j$ run from 1 to $d$ and the index after a comma represents the partial derivative with respect to the corresponding component of the independent variable. Summation over repeated indices is implied. We denote the divergence operator by Div $\sigma=\left(\sigma_{i j, j}\right)$. The standard Lebesgue and Sobolev spaces $L^{2}(\Omega)^{d}=L^{2}\left(\Omega ; \mathbb{R}^{d}\right)$ and $H^{1}(\Omega)^{d}=H^{1}\left(\Omega ; \mathbb{R}^{d}\right)$ are used. The linearized (small) strain tensor for displacement $\boldsymbol{u} \in H^{1}(\Omega)^{d}$ is defined by

$$
\boldsymbol{\varepsilon}(\boldsymbol{u})=\left(\varepsilon_{i j}(\boldsymbol{u})\right), \quad \varepsilon_{i j}(\boldsymbol{u})=\frac{1}{2}\left(u_{i, j}+u_{j, i}\right) .
$$

Let $u_{v}=\boldsymbol{u} \cdot \boldsymbol{v}$ and $\sigma_{v}=\sigma \boldsymbol{v} \cdot \boldsymbol{v}$ be the normal components of $\boldsymbol{u}$ and $\boldsymbol{\sigma}$, respectively, and let $\boldsymbol{u}_{\tau}=\boldsymbol{u}-u_{v} \boldsymbol{v}$ and $\sigma_{\tau}=\sigma \boldsymbol{v}-\sigma_{\nu} \boldsymbol{v}$ be their tangential components, respectively. In what follows, for simplicity, we sometimes do not indicate explicitly the dependence of various functions on the spatial variable $\boldsymbol{x}$.

Now let us introduce the classical formulation of considered mechanical contact problem.

Problem P: Find a displacement field $\boldsymbol{u}: \Omega \rightarrow \mathbb{R}^{d}$ and a stress field $\sigma: \Omega \rightarrow \mathbb{S}^{d}$ such that

$$
\begin{aligned}
\boldsymbol{\sigma}=\mathcal{A}(\boldsymbol{\varepsilon}(\boldsymbol{u})) & \text { in } \Omega \\
\operatorname{Div} \boldsymbol{\sigma}+\boldsymbol{f}_{0}=\mathbf{0} & \text { in } \Omega \\
\boldsymbol{u}=\mathbf{0} & \text { on } \Gamma_{D} \\
\boldsymbol{\sigma} \boldsymbol{v}=\boldsymbol{f}_{N} & \text { on } \Gamma_{N} \\
-\sigma_{v} \in \partial j_{v}\left(u_{v}\right) & \text { on } \Gamma_{C} \\
-\boldsymbol{\sigma}_{\boldsymbol{\tau}} \in h_{\tau}\left(u_{v}\right) \partial j_{\tau}\left(\boldsymbol{u}_{\boldsymbol{\tau}}\right) & \text { on } \Gamma_{C}
\end{aligned}
$$

Here, Eq. (4.1) represents an elastic constitutive law and $\mathcal{A}$ is an elasticity operator. Equilibrium equation (4.2) reflects the fact that problem is static. Equation (4.3) represents clamped boundary condition on $\Gamma_{D}$ and (4.4) represents the action of the traction on $\Gamma_{N}$. Inclusion (4.5) describes the response of the foundation in normal direction, whereas the friction is modeled by inclusion (4.6), where $j_{v}$ and $j_{\tau}$ are given superpotentials, and $h_{\tau}$ is a given friction bound.

We consider the following Hilbert spaces

$$
\mathcal{H}=L^{2}\left(\Omega ; \mathbb{S}^{d}\right), \quad V=\left\{\boldsymbol{v} \in H^{1}(\Omega)^{d} \mid \boldsymbol{v}=\mathbf{0} \text { on } \Gamma_{D}\right\}
$$

endowed with the inner scalar products

$$
(\boldsymbol{\sigma}, \boldsymbol{\tau})_{\mathcal{H}}=\int_{\Omega} \sigma_{i j} \tau_{i j} d x, \quad(\boldsymbol{u}, \boldsymbol{v})_{V}=(\boldsymbol{\varepsilon}(\boldsymbol{u}), \boldsymbol{\varepsilon}(\boldsymbol{v}))_{\mathcal{H}},
$$


respectively. The fact that space $V$ equipped with the norm $\|\cdot\|_{V}$ is complete follows from Korn's inequality, and its application is allowed because we assume that meas $\left(\Gamma_{D}\right)>0$. We consider the trace operator $\gamma: V \rightarrow L^{2}\left(\Gamma_{C}\right)^{d}=X$. By the Sobolev trace theorem we know that $\gamma \in \mathcal{L}(V, X)$ with the norm equal to $c_{\gamma}$.

Now we present the hypotheses on data of Problem $P$.

$\underline{H(\mathcal{A})}: \mathcal{A}: \Omega \times \mathbb{S}^{d} \rightarrow \mathbb{S}^{d}$ satisfies

(a) $\mathcal{A}(\boldsymbol{x}, \boldsymbol{\tau})=\left(a_{i j k h}(\boldsymbol{x}) \tau_{k h}\right)$ for all $\boldsymbol{\tau} \in \mathbb{S}^{d}$, a.e. $\boldsymbol{x} \in \Omega, a_{i j k h} \in L^{\infty}(\Omega)$,

(b) $\mathcal{A}\left(\boldsymbol{x}, \boldsymbol{\tau}_{1}\right) \cdot \boldsymbol{\tau}_{2}=\boldsymbol{\tau}_{1} \cdot \mathcal{A}\left(\boldsymbol{x}, \boldsymbol{\tau}_{2}\right)$ for all $\boldsymbol{\tau}_{1}, \boldsymbol{\tau}_{2} \in \mathbb{S}^{d}$, a.e. $\boldsymbol{x} \in \Omega$,

(c) there exists $m_{\mathcal{A}}>0$ such that $\mathcal{A}(\boldsymbol{x}, \boldsymbol{\tau}) \cdot \boldsymbol{\tau} \geq m_{\mathcal{A}}\|\boldsymbol{\tau}\|^{2}$ for all $\boldsymbol{\tau} \in \mathbb{S}^{d}$, a.e. $\boldsymbol{x} \in \Omega$.

$\underline{H\left(j_{\nu}\right)}: \quad j_{v}: \Gamma_{C} \times \mathbb{R} \rightarrow \mathbb{R}$ satisfies

(a) $j_{\nu}(\cdot, \xi)$ is measurable on $\Gamma_{C}$ for all $\xi \in \mathbb{R}$ and there exists $e \in L^{2}\left(\Gamma_{C}\right)$ such that $j_{v}(\cdot, e(\cdot)) \in L^{1}\left(\Gamma_{C}\right)$,

(b) $j_{v}(\boldsymbol{x}, \cdot)$ is locally Lipschitz continuous on $\mathbb{R}$ for a.e. $\boldsymbol{x} \in \Gamma_{C}$,

(c) there exist $c_{\nu 0}, c_{\nu 1} \geq 0$ such that

$$
\left|\partial_{2} j_{v}(\boldsymbol{x}, \xi)\right| \leq c_{\nu 0}+c_{\nu 1}|\xi| \text { for all } \xi \in \mathbb{R} \text {, a.e. } \boldsymbol{x} \in \Gamma_{C}
$$

(d) there exists $\alpha_{v} \geq 0$ such that

$$
\left(j_{v}\right)_{2}^{0}\left(\boldsymbol{x}, \xi_{1} ; \xi_{2}-\xi_{1}\right)+\left(j_{v}\right)_{2}^{0}\left(\boldsymbol{x}, \xi_{2} ; \xi_{1}-\xi_{2}\right) \leq \alpha_{v}\left|\xi_{1}-\xi_{2}\right|^{2}
$$

for all $\xi_{1}, \xi_{2} \in \mathbb{R}$, a.e. $\boldsymbol{x} \in \Gamma_{C}$.

$\underline{H\left(j_{\tau}\right)}: \quad j_{\tau}: \Gamma_{C} \times \mathbb{R}^{d} \rightarrow \mathbb{R}$ satisfies

(a) $j_{\tau}(\cdot, \xi)$ is measurable on $\Gamma_{C}$ for all $\boldsymbol{\xi} \in \mathbb{R}^{d}$ and there exists $\boldsymbol{e} \in L^{2}\left(\Gamma_{C}\right)^{d}$ such that $j_{\tau}(\cdot, \boldsymbol{e}(\cdot)) \in L^{1}\left(\Gamma_{C}\right)$,

(b) there exists $c_{\tau}>0$ such that

$$
\left|j_{\tau}\left(\boldsymbol{x}, \boldsymbol{\xi}_{1}\right)-j_{\tau}\left(\boldsymbol{x}, \boldsymbol{\xi}_{2}\right)\right| \leq c_{\tau}\left\|\boldsymbol{\xi}_{1}-\boldsymbol{\xi}_{2}\right\| \text { for all } \boldsymbol{\xi}_{1}, \boldsymbol{\xi}_{2} \in \mathbb{R}^{d} \text {, a.e. } \boldsymbol{x} \in \Gamma_{C}
$$

(c) there exists $\alpha_{\tau} \geq 0$ such that

$$
\left(j_{\tau}\right)_{2}^{0}\left(\boldsymbol{x}, \boldsymbol{\xi}_{1} ; \boldsymbol{\xi}_{2}-\boldsymbol{\xi}_{1}\right)+\left(j_{\tau}\right)_{2}^{0}\left(\boldsymbol{x}, \boldsymbol{\xi}_{2} ; \boldsymbol{\xi}_{1}-\boldsymbol{\xi}_{2}\right) \leq \alpha_{\tau}\left\|\boldsymbol{\xi}_{1}-\boldsymbol{\xi}_{2}\right\|^{2}
$$

for all $\boldsymbol{\xi}_{1}, \boldsymbol{\xi}_{2} \in \mathbb{R}^{d}$, a.e. $\boldsymbol{x} \in \Gamma_{C}$.

$\underline{H(h)}: \quad h_{\tau}: \Gamma_{C} \times \mathbb{R} \rightarrow \mathbb{R}$ satisfies

(a) $h_{\tau}(\cdot, \eta)$ is measurable on $\Gamma_{C}$ for all $\eta \in \mathbb{R}$,

(b) there exists $\overline{h_{\tau}}>0$ such that $0 \leq h_{\tau}(\boldsymbol{x}, \eta) \leq \overline{h_{\tau}}$ for all $\eta \in \mathbb{R}$, a.e. $\boldsymbol{x} \in \Gamma_{C}$,

(c) there exists $L_{h_{\tau}}>0$ such that 


$$
\left|h_{\tau}\left(\boldsymbol{x}, \eta_{1}\right)-h_{\tau}\left(\boldsymbol{x}, \eta_{2}\right)\right| \leq L_{h_{\tau}}\left|\eta_{1}-\eta_{2}\right| \text { for all } \eta_{1}, \eta_{2} \in \mathbb{R} \text {, a.e. } \boldsymbol{x} \in \Gamma_{C} \text {. }
$$

$\left(\underline{\left.H_{0}\right)}: \quad \boldsymbol{f}_{0} \in L^{2}(\Omega)^{d}, \quad \boldsymbol{f}_{N} \in L^{2}\left(\Gamma_{N}\right)^{d}\right.$.

We remark that condition $H\left(j_{\tau}\right)(\mathrm{b})$ is equivalent to the fact that $j_{\tau}(\boldsymbol{x}, \cdot)$ is locally Lipschitz continuous and there exists $c_{\tau} \geq 0$ such that $\left\|\partial_{2} j_{\tau}(\boldsymbol{x}, \boldsymbol{\xi})\right\| \leq c_{\tau}$ for all $\xi \in \mathbb{R}^{d}$ and a.e. $\boldsymbol{x} \in \Gamma_{C}$. Moreover, condition $H(h)(\mathrm{b})$ is sufficient to obtain presented mathematical results, but from mechanical point of view we should additionally assume that $h(r)=0$ for $r \leq 0$. This corresponds to the situation when body is separated from the foundation and friction force vanishes.

Using the standard procedure, the Green formula and the definition of generalized subdifferential, we obtain a weak formulation of Problem $P$ in the form of hemivariational inequality.

Problem $\boldsymbol{P}_{\boldsymbol{h v i}}$ Find a displacement $\boldsymbol{u} \in V$ such that for all $\boldsymbol{v} \in V$

$$
\langle A \boldsymbol{u}, \boldsymbol{v}\rangle_{V^{*} \times V}+\int_{\Gamma_{C}} j_{3}^{0}(\boldsymbol{x}, \gamma \boldsymbol{u}(\boldsymbol{x}), \gamma \boldsymbol{u}(\boldsymbol{x}) ; \gamma \boldsymbol{v}(\boldsymbol{x})) d a \geq\langle\boldsymbol{f}, \boldsymbol{v}\rangle_{V^{*} \times V}
$$

Here, the operator $A: V \rightarrow V^{*}$ and $\boldsymbol{f} \in V^{*}$ are defined for all $\boldsymbol{w}, \boldsymbol{v} \in V$ as follows

$$
\begin{aligned}
\langle A \boldsymbol{w}, \boldsymbol{v}\rangle_{V^{*} \times V} & =(\mathcal{A}(\boldsymbol{\varepsilon}(\boldsymbol{w})), \boldsymbol{\varepsilon}(\boldsymbol{v}))_{\mathcal{H}} \\
\langle\boldsymbol{f}, \boldsymbol{v}\rangle_{V^{*} \times V} & =\int_{\Omega} \boldsymbol{f}_{0} \cdot \boldsymbol{v} d x+\int_{\Gamma_{N}} \boldsymbol{f}_{N} \cdot \gamma \boldsymbol{v} d a
\end{aligned}
$$

and $j: \Gamma_{C} \times \mathbb{R}^{d} \times \mathbb{R}^{d} \rightarrow \mathbb{R}$ is defined for all $\eta, \xi \in \mathbb{R}^{d}$ and $\boldsymbol{x} \in \Gamma_{C}$ by

$$
j(\boldsymbol{x}, \boldsymbol{\eta}, \boldsymbol{\xi})=j_{v}\left(\boldsymbol{x}, \xi_{v}\right)+h_{\tau}\left(\boldsymbol{x}, \eta_{v}\right) j_{\tau}\left(\boldsymbol{x}, \boldsymbol{\xi}_{\tau}\right)
$$

It is easy to check that under assumptions $H(\mathcal{A})$ and $\left(H_{0}\right)$, the operator $A$ and the functional $f$ satisfy $H(A)$ and $H(f)$, respectively. We also define the functional $J: L^{2}\left(\Gamma_{C}\right)^{d} \times L^{2}\left(\Gamma_{C}\right)^{d} \rightarrow \mathbb{R}$ for all $\boldsymbol{w}, \boldsymbol{v} \in L^{2}\left(\Gamma_{C}\right)^{d}$ by

$$
J(\boldsymbol{w}, \boldsymbol{v})=\int_{\Gamma_{C}} j(\boldsymbol{x}, \boldsymbol{w}(\boldsymbol{x}), \boldsymbol{v}(\boldsymbol{x})) d a .
$$

Below we present some properties of the functional $J$.

Lemma 6 Assumptions $H\left(j_{v}\right), H\left(j_{\tau}\right)$ and $H(h)$ imply that functional $J$ defined by (4.8)-(4.9) satisfies $H(J)$.

Proof We first observe that from $H\left(j_{v}\right)(\mathrm{a}),(\mathrm{b}), H\left(j_{\tau}\right)(\mathrm{a}),(\mathrm{b})$ and $H(h)(\mathrm{a}),(\mathrm{c})$ the function $j(\cdot, \boldsymbol{\eta}, \boldsymbol{\xi})$ is measurable on $\Gamma_{C}$, there exist $\boldsymbol{e}_{1}, \boldsymbol{e}_{2} \in L^{2}\left(\Gamma_{C}\right)^{d}$ such that $j\left(\cdot, \boldsymbol{e}_{1}(\cdot), \boldsymbol{e}_{2}(\cdot)\right) \in L^{1}\left(\Gamma_{C}\right), j(\boldsymbol{x}, \cdot, \boldsymbol{\xi})$ is continuous and $j(\boldsymbol{x}, \boldsymbol{\eta}, \cdot)$ is locally Lipschitz. Moreover, by $H\left(j_{v}\right)(\mathrm{c}), H\left(j_{\tau}\right)(\mathrm{c})$ and $H(h)(\mathrm{b})$ we easily conclude

$\left\|\partial_{3} j(\boldsymbol{x}, \boldsymbol{\eta}, \boldsymbol{\xi})\right\| \leq\left|\partial_{2} j_{v}\left(\boldsymbol{x}, \xi_{v}\right)\right|+h_{\tau}\left(\boldsymbol{x}, \eta_{v}\right)\left\|\partial_{2} j_{\tau}\left(\boldsymbol{x}, \boldsymbol{\xi}_{\tau}\right)\right\| \leq c_{\nu 0}+\left(c_{\nu 1}+\overline{h_{\tau}} c_{\tau}\right)\|\boldsymbol{\xi}\|$. 
Applying Corollary 4.15 in [15], we obtain that functional $J$ is well defined, locally Lipschitz with respect to the second variable and the growth condition $H(J)(\mathrm{b})$ holds with $c_{0}=\sqrt{2 \operatorname{meas}\left(\Gamma_{C}\right)} c_{\nu 0}, c_{1}=\sqrt{2}\left(c_{\nu 1}+\overline{h_{\tau}} c_{\tau}\right)$ and $c_{2}=0$.

To prove $H(J)(\mathrm{c})$, we take $\boldsymbol{\eta}_{i}, \boldsymbol{\xi}_{i} \in \mathbb{R}^{d}, i=1,2$, and by the sum rules (cf. Proposition 3.35 in [15]) and from $H\left(j_{v}\right)(\mathrm{d}), H\left(j_{\tau}\right)(\mathrm{b}),(\mathrm{c})$ and $H(h)(\mathrm{b}),(\mathrm{c})$, we obtain

$$
\begin{aligned}
& j_{3}^{0}\left(\boldsymbol{x}, \boldsymbol{\eta}_{1}, \boldsymbol{\xi}_{1} ; \boldsymbol{\xi}_{2}-\boldsymbol{\xi}_{1}\right)+j_{3}^{0}\left(\boldsymbol{x}, \boldsymbol{\eta}_{2}, \boldsymbol{\xi}_{2} ; \boldsymbol{\xi}_{1}-\boldsymbol{\xi}_{2}\right) \\
& \quad \leq\left(j_{v}\right)_{2}^{0}\left(\boldsymbol{x}, \xi_{1 v} ; \xi_{2 v}-\xi_{1 v}\right)+\left(j_{v}\right)_{2}^{0}\left(\boldsymbol{x}, \xi_{2 v} ; \xi_{1 v}-\xi_{2 v}\right) \\
& \quad+h_{\tau}\left(\boldsymbol{x}, \eta_{1 v}\right)\left(\left(j_{\tau}\right)_{2}^{0}\left(\boldsymbol{x}, \boldsymbol{\xi}_{1 \tau} ; \boldsymbol{\xi}_{2 \tau}-\boldsymbol{\xi}_{1 \tau}\right)+\left(j_{\tau}\right)_{2}^{0}\left(\boldsymbol{x}, \boldsymbol{\xi}_{2 \tau} ; \boldsymbol{\xi}_{1 \tau}-\boldsymbol{\xi}_{2 \tau}\right)\right) \\
& \quad+\left(h_{\tau}\left(\boldsymbol{x}, \eta_{2 v}\right)-h_{\tau}\left(\boldsymbol{x}, \eta_{1 v}\right)\right)\left(j_{\tau}\right)_{2}^{0}\left(\boldsymbol{x}, \boldsymbol{\xi}_{2 \tau} ; \boldsymbol{\xi}_{1 \tau}-\boldsymbol{\xi}_{2 \tau}\right) \\
& \quad \leq\left(\alpha_{v}+\overline{h_{\tau}} \alpha_{\tau}\right)\left\|\boldsymbol{\xi}_{1}-\boldsymbol{\xi}_{2}\right\|^{2}+L_{h_{\tau}} c_{\tau}\left\|\boldsymbol{\eta}_{1}-\boldsymbol{\eta}_{2}\right\|\left\|\boldsymbol{\xi}_{1}-\boldsymbol{\xi}_{2}\right\| .
\end{aligned}
$$

And consequently, since

$$
J_{2}^{0}(\boldsymbol{w}, \boldsymbol{v} ; \boldsymbol{z}) \leq \int_{\Gamma_{C}} j_{3}^{0}(\boldsymbol{x}, \boldsymbol{w}(\boldsymbol{x}), \boldsymbol{v}(\boldsymbol{x}) ; \boldsymbol{z}(\boldsymbol{x})) d a
$$

(cf. Corollary 4.15(iii) in [15]), we have

$$
\begin{aligned}
& J_{2}^{0}\left(\boldsymbol{w}_{1}, \boldsymbol{v}_{1} ; \boldsymbol{v}_{2}-\boldsymbol{v}_{1}\right)+J_{2}^{0}\left(\boldsymbol{w}_{2}, \boldsymbol{v}_{2} ; \boldsymbol{v}_{1}-\boldsymbol{v}_{2}\right) \\
& \quad \leq \int_{\Gamma_{C}}\left(\left(\alpha_{v}+\overline{h_{\tau}} \alpha_{\tau}\right)\left\|\boldsymbol{v}_{1}(\boldsymbol{x})-\boldsymbol{v}_{2}(\boldsymbol{x})\right\|^{2}+L_{h_{\tau}} c_{\tau}\left\|\boldsymbol{w}_{1}(\boldsymbol{x})-\boldsymbol{w}_{2}(\boldsymbol{x})\right\|\left\|\boldsymbol{v}_{1}(\boldsymbol{x})-\boldsymbol{v}_{2}(\boldsymbol{x})\right\|\right) d a
\end{aligned}
$$

Hence, by the Hölder inequality, we obtain $H(J)(\mathrm{c})$ with $m_{\alpha}=\alpha_{v}+\overline{h_{\tau}} \alpha_{\tau}$ and $m_{L}=L_{h_{\tau}} c_{\tau}$.

With the above properties, we have the following existence and uniqueness result for Problem $P_{h v i}$.

Theorem 7 If assumptions $H(\mathcal{A}), H\left(j_{v}\right), H\left(j_{\tau}\right), H(h),\left(H_{0}\right)$ and $\left(H_{S}\right)$ hold, then Problems $P_{\text {hvi }}$ and $P_{\text {incl }}$ are equivalent. Moreover, they have a unique solution $\boldsymbol{u} \in V$ and this solution satisfies

$$
\|\boldsymbol{u}\|_{V} \leq c\left(1+\|\boldsymbol{f}\|_{V^{*}}\right)
$$

with a positive constant $c$.

Proof We notice that the assumptions of Theorem 4 are satisfied. This implies that Problem $P_{\text {incl }}$ has a unique solution. By (2.4) and Corollary 4.15(iii) in [15] we get that every solution to Problem $P_{i n c l}$ solves Problem $P_{h v i}$. Using similar technique as in the proof of Lemma 1, we can show that if Problem $P_{h v i}$ has a solution, it is unique. Combining these facts we obtain our assertion.

We conclude this section by providing a sample error estimate under additional assumptions on the solution regularity. We consider a polygonal domain $\Omega$ and a space 
of continuous piecewise affine functions $V^{h}$. We introduce the following discretized version of Problem $P_{h v i}$.

Problem $\boldsymbol{P}_{h v i}^{h}$ Find a displacement $\boldsymbol{u}^{h} \in V^{h}$ such that for all $\boldsymbol{v}^{h} \in V^{h}$

$$
\left\langle A \boldsymbol{u}^{h}, \boldsymbol{v}^{h}\right\rangle_{V^{*} \times V}+\int_{\Gamma_{C}} j_{3}^{0}\left(\boldsymbol{x}, \gamma \boldsymbol{u}^{h}(\boldsymbol{x}), \gamma \boldsymbol{u}^{h}(\boldsymbol{x}) ; \gamma \boldsymbol{v}^{h}(\boldsymbol{x})\right) d a \geq\left\langle\boldsymbol{f}, \boldsymbol{v}^{h}\right\rangle_{V^{*} \times V} .
$$

Theorem 8 Assume $H(\mathcal{A}), H\left(j_{v}\right), H\left(j_{\tau}\right), H(h),\left(H_{0}\right)$ and $\left(H_{S}\right)$ and assume the solution regularity $\boldsymbol{u} \in H^{2}(\Omega)^{d}, \gamma \boldsymbol{u} \in H^{2}\left(\Gamma_{C}\right)^{d}, \boldsymbol{\sigma} \boldsymbol{v} \in L^{2}\left(\Gamma_{C}\right)^{d}$. Additionally, assume that $\Gamma_{C}$ is a flat component of the boundary $\Gamma$. Then, for the solution $\boldsymbol{u}$ to Problem $P_{h v i}$ and the solution $\boldsymbol{u}^{h}$ to Problem $P_{\text {hvi }}^{h}$ there exists a constant $c>0$ such that

$$
\left\|\boldsymbol{u}-\boldsymbol{u}^{h}\right\|_{V} \leq c h
$$

Proof We denote by $\Pi^{h} \boldsymbol{u} \in V^{h}$ the finite element interpolant of $\boldsymbol{u}$. By the standard finite element interpolation error bounds (see [5]) we have for all $\eta \in H^{2}(\Omega)^{d}$ such that $\gamma \eta \in H^{2}\left(\Gamma_{C}\right)^{d}$

$$
\begin{gathered}
\left\|\boldsymbol{\eta}-\Pi^{h} \eta\right\|_{V} \leq c h\|\boldsymbol{\eta}\|_{H^{2}(\Omega)^{d}}, \\
\left\|\gamma \boldsymbol{\eta}-\gamma \Pi^{h} \eta\right\|_{L^{2}\left(\Gamma_{C}\right)^{d}} \leq c h^{2}\|\gamma \boldsymbol{\eta}\|_{H^{2}\left(\Gamma_{C}\right)^{d}} .
\end{gathered}
$$

We now bound the residual term defined by (3.2) using similar procedure to that one described in [8]. Let $\boldsymbol{v}= \pm \boldsymbol{w}$ in inequality (4.7), where the arbitrary function $\boldsymbol{w} \in V$ is such that $\boldsymbol{w} \in C^{\infty}(\bar{\Omega})^{d}$ and $\boldsymbol{w}=\mathbf{0}$ on $\Gamma_{D} \cup \Gamma_{C}$. Then we obtain the identity

$$
\langle A \boldsymbol{u}, \boldsymbol{w}\rangle_{V^{*} \times V}=\langle\boldsymbol{f}, \boldsymbol{w}\rangle_{V^{*} \times V}
$$

From this identity, using fundamental lemma of calculus of variations, we can deduce that

$$
\begin{array}{rlrl}
\operatorname{Div} \mathcal{A}(\boldsymbol{\varepsilon}(\boldsymbol{u}))+\boldsymbol{f}_{0} & =\mathbf{0} & \text { in } \Omega, \\
\boldsymbol{\sigma} \boldsymbol{v} & =\boldsymbol{f}_{N} & & \text { on } \Gamma_{N} .
\end{array}
$$

We multiply equation (4.13) by $\boldsymbol{v}^{h}-\boldsymbol{u}$ and obtain

$$
\int_{\Gamma} \boldsymbol{\sigma} \boldsymbol{v} \cdot\left(\gamma \boldsymbol{v}^{h}-\gamma \boldsymbol{u}\right) d a-\int_{\Omega} \mathcal{A}(\boldsymbol{\varepsilon}(\boldsymbol{u})) \cdot \boldsymbol{\varepsilon}\left(\boldsymbol{v}^{h}-\boldsymbol{u}\right) d x+\int_{\Omega} \boldsymbol{f}_{0} \cdot\left(\boldsymbol{v}^{h}-\boldsymbol{u}\right) d x=0 .
$$

Using the homogenous Dirichlet boundary condition of $\boldsymbol{v}^{h}-\boldsymbol{u}$ on $\Gamma_{D}$ and the traction boundary condition given by (4.14) we have

$$
\left\langle A \boldsymbol{u}, \boldsymbol{v}^{h}-\boldsymbol{u}\right\rangle_{V^{*} \times V}=\int_{\Gamma_{C}} \boldsymbol{\sigma} \boldsymbol{v} \cdot\left(\gamma \boldsymbol{v}^{h}-\gamma \boldsymbol{u}\right) d a+\left\langle\boldsymbol{f}, \boldsymbol{v}^{h}-\boldsymbol{u}\right\rangle_{V^{*} \times V}
$$


Using this and (3.2) we obtain

$$
R\left(\boldsymbol{u}, \boldsymbol{v}^{h}\right)=\int_{\Gamma_{C}} \boldsymbol{\sigma} \boldsymbol{v} \cdot\left(\gamma \boldsymbol{v}^{h}-\gamma \boldsymbol{u}\right) d a \leq c\left\|\gamma \boldsymbol{u}-\gamma \boldsymbol{v}^{h}\right\|_{L^{2}\left(\Gamma_{C}\right)^{d}} .
$$

From inequalities (3.1), (4.11), (4.12) and (4.17) we get

$$
\left\|\boldsymbol{u}-\boldsymbol{u}^{h}\right\|_{V}^{2} \leq c\left(\left\|\boldsymbol{u}-\Pi^{h} \boldsymbol{u}\right\|_{V}^{2}+\left\|\gamma \boldsymbol{u}-\gamma \Pi^{h} \boldsymbol{u}\right\|_{L^{2}\left(\Gamma_{C}\right)^{d}}\right) \leq c h^{2},
$$

and we obtain required estimate.

\section{Simulations}

In this section we present results of our computational simulations. From Theorems 4 and 7 we know that Problems $P_{h v i}$ and $P_{o p t}$ are equivalent. Hence, we can apply numerical scheme $P_{o p t}^{h}$ and use Theorem 5 to approximate solution of $P_{h v i}$. We employ Finite Element Method and use space $V^{h}$ of continuous piecewise affine functions as a family of approximating subspaces. The idea for algorithm used to calculate solution of discretized problem is based on the proof of Lemma 3 and is described by Algorithm 1.

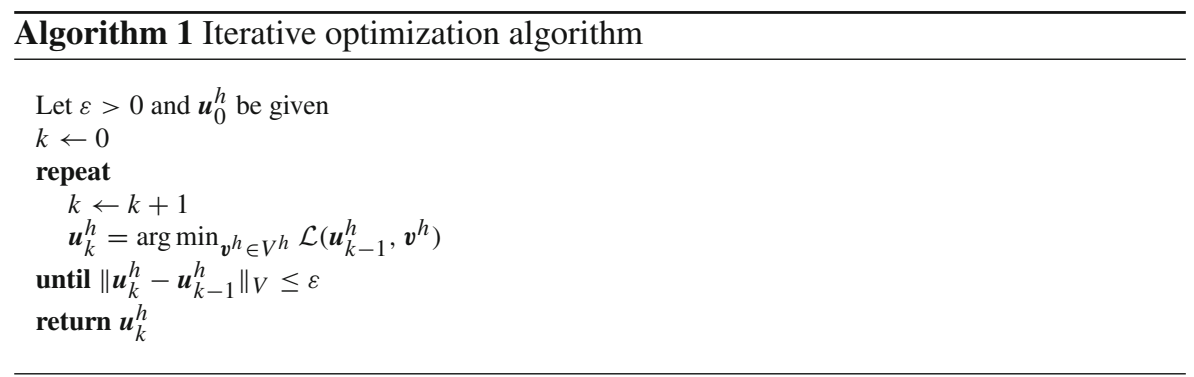

In order to minimize not necessarily differentiable function $\mathcal{L}\left(\boldsymbol{w}^{h}, \cdot\right)$ we use Powell's conjugate direction method. This method was introduced in [18] and does not require the assumption on differentiability of optimized function. Other, more refined nonsmooth optimization algorithms described for example in [1], could also be adapted. For a starting point $\boldsymbol{u}_{0}^{h}$ we take a solution to problem with $\boldsymbol{\sigma} \boldsymbol{v}=\mathbf{0}$ on $\Gamma_{C}$, although it can be chosen arbitrarily.

We set $d=2$ and consider a rectangular set $\Omega=[0,2] \times[0,1]$ with following parts of the boundary

$$
\Gamma_{D}=\{0\} \times[0,1], \quad \Gamma_{N}=([0,2] \times\{1\}) \cup(\{2\} \times[0,1]), \quad \Gamma_{C}=[0,2] \times\{0\} .
$$

The elasticity operator $\mathcal{A}$ is defined by

$$
\mathcal{A}(\boldsymbol{\tau})=2 \eta \boldsymbol{\tau}+\lambda \operatorname{tr}(\boldsymbol{\tau}) I, \quad \boldsymbol{\tau} \in \mathbb{S}^{2} .
$$




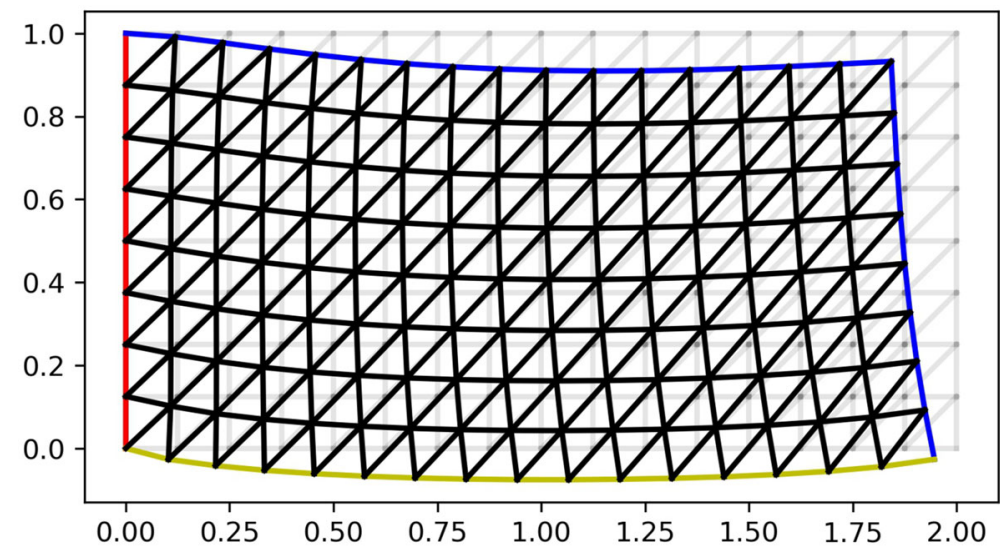

Fig. 1 Initial data

Here $I$ denotes the identity matrix, tr denotes the trace of the matrix, $\lambda$ and $\eta$ are the Lamé coefficients, $\lambda, \eta>0$. In our simulations we take the following data

$$
\begin{aligned}
& \lambda=\eta=4, \\
& \boldsymbol{u}_{0}(\boldsymbol{x})=(0,0), \quad \boldsymbol{x} \in \Omega, \\
& j_{v}(x, \xi)= \begin{cases}0, & \xi \in(-\infty, 0), \\
10 \xi^{2}, & \xi \in[0,0.1), \quad x \in \Gamma_{C}, \\
0.1, & \xi \in[0.1, \infty),\end{cases} \\
& j_{\tau}(\boldsymbol{x}, \boldsymbol{\xi})=\ln (\|\boldsymbol{\xi}\|+1), \quad \boldsymbol{\xi} \in \mathbb{R}^{2}, \boldsymbol{x} \in \Gamma_{C}, \\
& h_{\tau}(\boldsymbol{x}, \eta)=\left\{\begin{array}{l}
0, \quad \eta \in(-\infty, 0), \\
8 \eta, \eta \in[0,0.1), \quad x \in \Gamma_{C}, \\
0.8 \quad \eta \in[0.1, \infty)
\end{array}\right. \\
& \boldsymbol{f}_{0}(\boldsymbol{x})=(-1.2,-0.9), \quad \boldsymbol{x} \in \Omega, \\
& \boldsymbol{f}_{N}(\boldsymbol{x})=(0,0), \quad \boldsymbol{x} \in \Omega \text {. }
\end{aligned}
$$

Both functions $j_{v}$ and $j_{\tau}$ are nondifferentiable and nonconvex. Our aim is to investigate reaction of the body to various modifications of input data.

In Fig. 1 we present output obtained without any modifications. We push the body down and to the left with a force $f_{0}$. As a result the body penetrates the foundation, but because of frictional forces it is squeezed to the left more in the higher part than in the lower part. In Fig. 2 we modify the function $h_{\tau}$ to be given by 


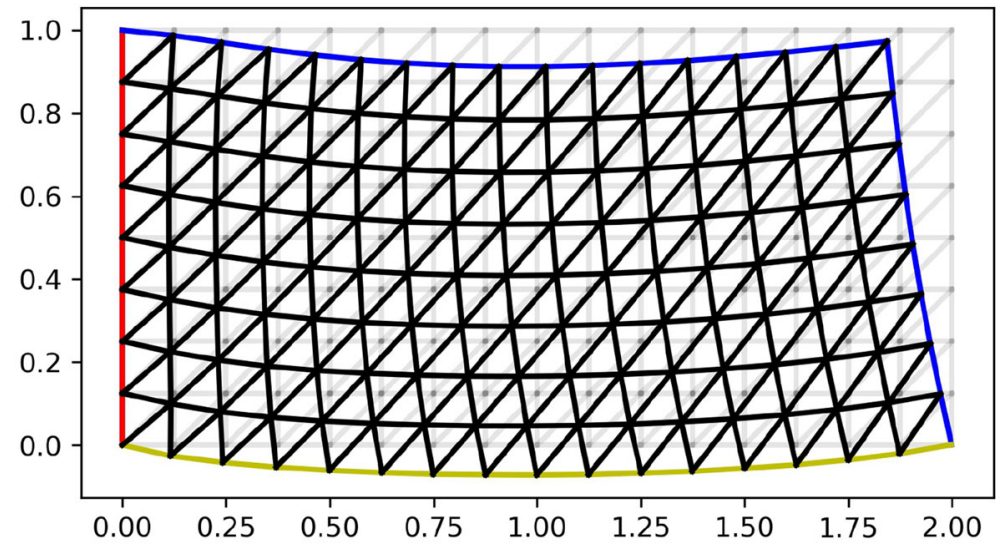

Fig. 2 Modified function $h_{\tau}$

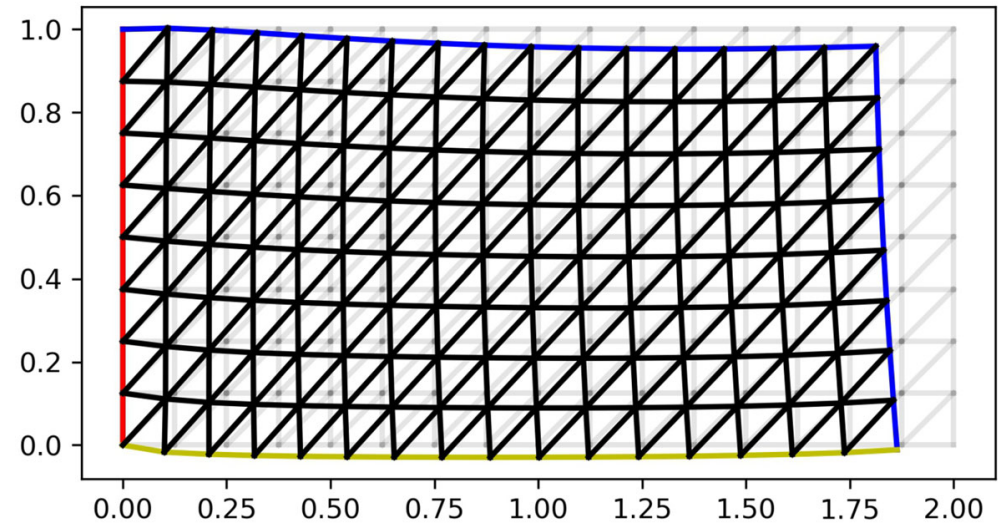

Fig. 3 Modified function $j_{v}$

$$
h_{\tau}(\boldsymbol{x}, \eta)= \begin{cases}0, & \eta \in(-\infty, 0), \\ 16 \eta, & \eta \in[0,0.1), \quad x \in \Gamma_{C} . \\ 1.6 & \eta \in[0.1, \infty)\end{cases}
$$

As a result we see that the penetration of the foundation does not change, but increased friction prevents the body from sliding to the left on $\Gamma_{C}$. In Fig. 3 we return to original data and modify only the function $j_{v}$ to the following

$$
j_{v}(x, \xi)= \begin{cases}0, & \xi \in(-\infty, 0), \\ 30 \xi^{2}, & \xi \in[0,0.1), \quad x \in \Gamma_{C} . \\ 0.3, & \xi \in[0.1, \infty),\end{cases}
$$




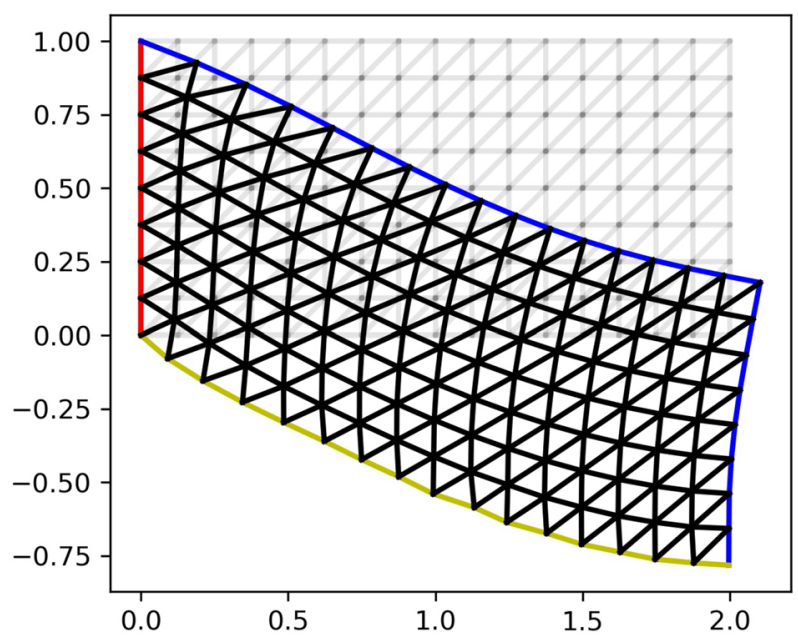

Fig. 4 Increased force $f_{0}$

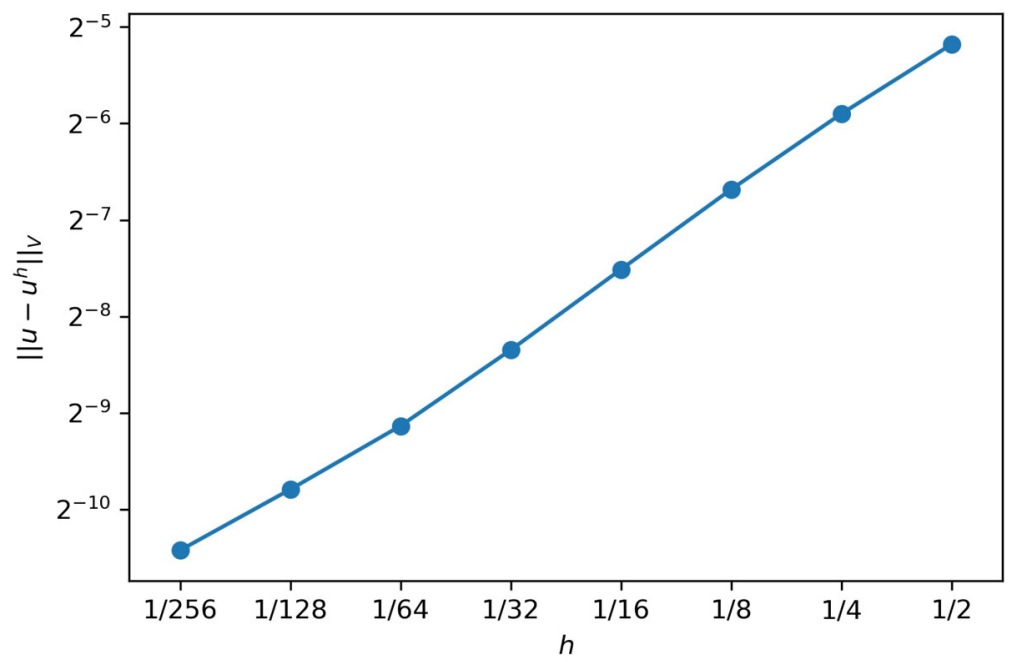

Fig. 5 Numerical errors

We can observe that the response of the foundation is more significant and the body moves downward only slightly. At the same time friction decreases due to influence of function $h_{\tau}$ that depends on normal component of body displacement. In Fig. 4 we once more return to original data and slightly increase force $f_{0}$ to be equal to

$$
\boldsymbol{f}_{0}(\boldsymbol{x})=(-1.2,-1.0), \quad \boldsymbol{x} \in \Omega \text {. }
$$

As a result, the body breaks through the threshold of quadratic response of the function $j_{v}$ into the part where this function is constant. This reflects the situation when there 
is no response of the foundation in normal direction (e.g. the foundation broke) and causes the penetration to increase drastically.

In order to illustrate the error estimate obtained in Sect. 4, we present a comparison of numerical errors $\left\|\boldsymbol{u}-\boldsymbol{u}^{h}\right\|_{V}$ computed for a sequence of solutions to discretized problems. We use a uniform discretization of the problem domain according to the spatial discretization parameter $h$. The boundary $\Gamma_{C}$ of $\Omega$ is divided into $1 / h$ equal parts. We start with $h=1$, which is successively halved. The numerical solution corresponding to $h=1 / 512$ was taken as the "exact" solution $\boldsymbol{u}$. The numerical results are presented in Fig. 5, where the dependence of the error estimate $\left\|\boldsymbol{u}-\boldsymbol{u}^{h}\right\|_{V}$ with respect to $h$ is plotted on a log-log scale. A first order convergence can be observed, providing numerical evidence of the theoretical optimal order error estimate obtained at the end of Sect. 4.

Acknowledgements The project leading to this application has received funding from the European Union's Horizon 2020 research and innovation programme under the Marie Sklodowska-Curie Grant Agreement No. 823731 CONMECH, National Science Center of Poland under Maestro Project No. UMO2012/06/A/ST1/00262.

\section{Compliance with Ethical Standards}

Conflict of interest The authors declare that they have no conflict of interest.

Open Access This article is distributed under the terms of the Creative Commons Attribution 4.0 International License (http://creativecommons.org/licenses/by/4.0/), which permits unrestricted use, distribution, and reproduction in any medium, provided you give appropriate credit to the original author(s) and the source, provide a link to the Creative Commons license, and indicate if changes were made.

\section{References}

1. Bagirov, A., Karmitsa, N., Mäkelä, M.M.: Introduction to Nonsmooth Optimization Theory, Practice and Software. Springer, New York (2014)

2. Barboteu, M., Bartosz, K., Kalita, P.: An analytical and numerical approach to a bilateral contact problem with nonmonotone friction. Int. J. Appl. Math. Comput. Sci. 23(2), 263-276 (2013)

3. Barboteu, M., Bartosz, K., Kalita, P., Ramadan, A.: Analysis of a contact problem with normal compliance, finite penetration and nonmonotone slip dependent friction. Commun. Contemp. Math. 16(1), 1350016 (2014)

4. Barboteu, M., Han, W., Migórski, S.: On numerical approximation of a variational-hemivariational inequality modeling contact problems for locking materials. Comput. Math. Appl. 77(11), 2894-2905 (2019)

5. Ciarlet, P.G.: The Finite Element Method for Elliptic Problems. North Holland, Amsterdam (1978)

6. Clarke, F.H.: Optimization and Nonsmooth Analysis. Wiley Interscience, New York (1983)

7. Fan, L., Liu, S., Gao, S.: Generalized monotonicity and convexity of non-differentiable functions. J. Math. Anal. Appl. 279, 276-289 (2003)

8. Han, W.: Numerical analysis of stationary variational-hemivariational inequalities with applications in contact mechanics. Math. Mech. Solids 1-15 (2017)

9. Han, W., Sofonea, M.: Numerical analysis of hemivariational inequalities in contact mechanics. Acta Numerica 28, 175-286 (2019)

10. Han, W., Migórski, S., Sofonea, M.: Advances in Variational and Hemivariational Inequalities, Advances in Mechanics and Mathematics, vol. 33. Springer, New York (2015)

11. Han, W., Sofonea, M., Barboteu, M.: Numerical analysis of elliptic hemivariational inequalities. SIAM J. Numer. Anal. 55(2), 640-663 (2017) 
12. Han, W., Sofonea, M., Danan, D.: Numerical analysis of stationary variational-hemivariational inequalities. Numer. Mathe. 139(3), 563-592 (2018)

13. Haslinger, J., Miettinen, M., Panagiotopoulos, P.D.: Finite Element Method for Hemivariational Inequalities. Theory, Methods and Applications. Kluwer Academic Publishers, Boston (1999)

14. Miettinen, M., Haslinger, J.: Finite element approximation of vector-valued hemivariational problems. J. Glob. Optim. 10(1), 17-35 (1997)

15. Migórski, S., Ochal, A., Sofonea, M.: Nonlinear Inclusions and Hemivariational Inequalities: Models and Analysis of Contact Problems, vol. 26. Springer, New York (2013)

16. Migórski, S., Ochal, A., Sofonea, M.: A class of variational-hemivariational inequalities in reflexive Banach spaces. J. Elast. 127(2), 151-178 (2017)

17. Panagiotopoulos, P.D.: Hemivariational Inequalities, Applications in Mechanics and Engineering. Springer, New York (1993)

18. Powell, M.J.D.: An efficient method for finding the minimum of a function of several variables without calculating derivatives. Comput. J. 7(2), 155-162 (1964)

19. Wriggers, P.: Computational Contact Mechanics. Wiley, Chichester (2002)

Publisher's Note Springer Nature remains neutral with regard to jurisdictional claims in published maps and institutional affiliations. 